\title{
Rare Case of Small Unilateral Dermoid Causing Anti-NMDAR (N-methyl- D Aspartate Receptor) Encephalitis
}

\author{
Natasha Gardiner* and Chukwumobi Ihezue
}

Queen Alexandra Hospital, Portsmouth, England

*Correspondence: Natasha Gardiner, Queen Alexandra Hospital, Portsmouth, England, E-mail: gardiner.natasha@gmail.com

\begin{abstract}
The association between ovarian teratomas and anti-NMDAR (N-methyl- D Aspartate Receptor) encephalitis is not commonly recognized. Diagnosis is based on the radiological appearances of fat containing teratomas on transvaginal or transabdominal ultrasounds, CT and MR scans, as well as blood tests to confirm the presence of specific antibodies. We present a rare case, which presented at our institution and discuss the presentation, investigations and treatments carried out.
\end{abstract}

Keywords: Ovarian teratomas; Anti-NMDAR encephalitis; Immunotherapy

Received Date: August 06, 2019; Accepted Date: August 14, 2019; Published Date: August 21, 2019

\section{Case Report}

An 18-year-old Brazilian female presented with a reduced GCS and worsening seizures and confusion. She was admitted to ITU. For 3 weeks prior to this admission she had been undergoing investigation for a recent history of headaches and worsening neurology. An MRI head and a CT head and venogram had been carried out and were all unremarkable.

The aetiology of her symptoms was unclear. She denied any trauma, recent travel, and ingestion of new foods or medications. It was thought perhaps a paraneoplastic cause or sources of antibodies for an autoimmune condition were to blame. A CT chest, abdomen and pelvis and a CT-PET were performed which both showed no obvious abnormality.

As the blood and radiological investigations obtained had provided negative results, a neurology review was requested. The consultant involved thought it prudent to test for NMDAR antibodies and review the current imaging again to see if any small dermoid tumours had been missed. A link between the NMDAR antibodies and teratomas, which resulted in similar clinical signs and symptoms, was thought a possible cause.

Blood was taken and tested for NMDAR antibodies. The results were found to be positive.

Citation: Natasha Gardiner, Rare Case of Small Unilateral Dermoid Causing Anti-NMDAR (N-methyl- D Aspartate Receptor) Encephalitis. J Clin Cases Rep 3(2): 43-47.

(C) 2020 Tridha Scholars 
The CT chest abdomen pelvis was reviewed by a consultant gynaecological radiologist. The Hounsfield unit values demonstrated small amounts of fat within the right ovary (Figure 1). A transvaginal ultrasound was advised and if nonconclusive an MRI should be performed.

The transvaginal ultrasound displayed a small area of fat reflectivity in the right ovary (Figure 2 and 3). This was reported as being non specific but had the potential to represent a dermoid. Following this an MRI pelvis depicted fat content in the right ovary (Figure 4 and 5) and so a right ovarian teratoma was diagnosed.

3 days following the MRI scan, the right ovary and teratoma were removed. The encephalitis subsequently improved and a repeat blood test for NMDAR antibodies revealed an equivocal result.

The patient returned to Brazil to be closer to her family, following almost complete recovery from the illness.

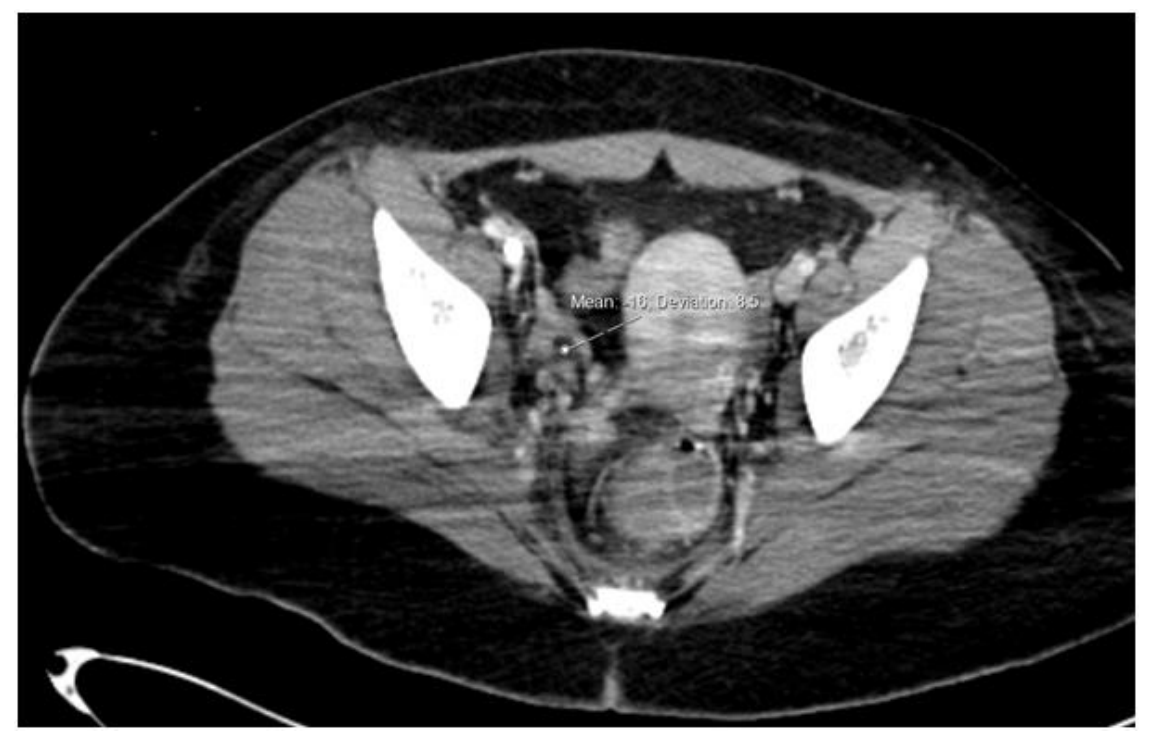

Figure 1: CT image showing HU-16, suggestive of fat content, in the right ovary.

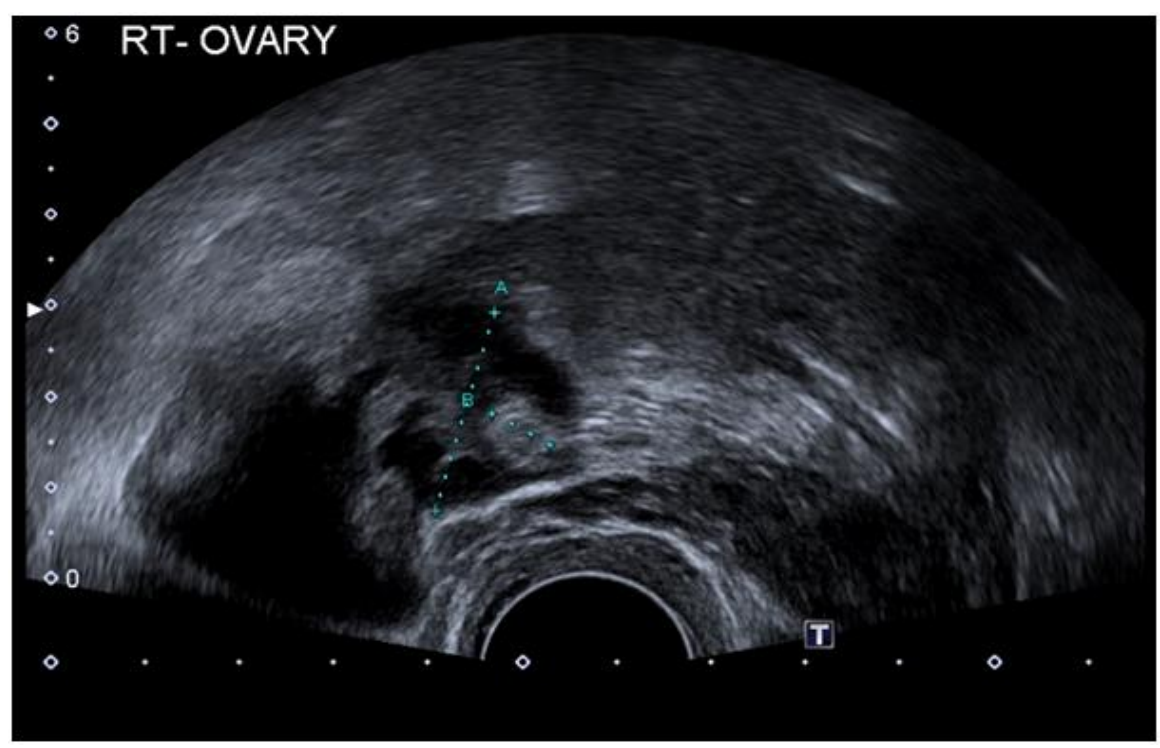

Figure 2: Ultrasound image depicting a small high reflectivity area, which is suggestive of fat in the right ovary. 


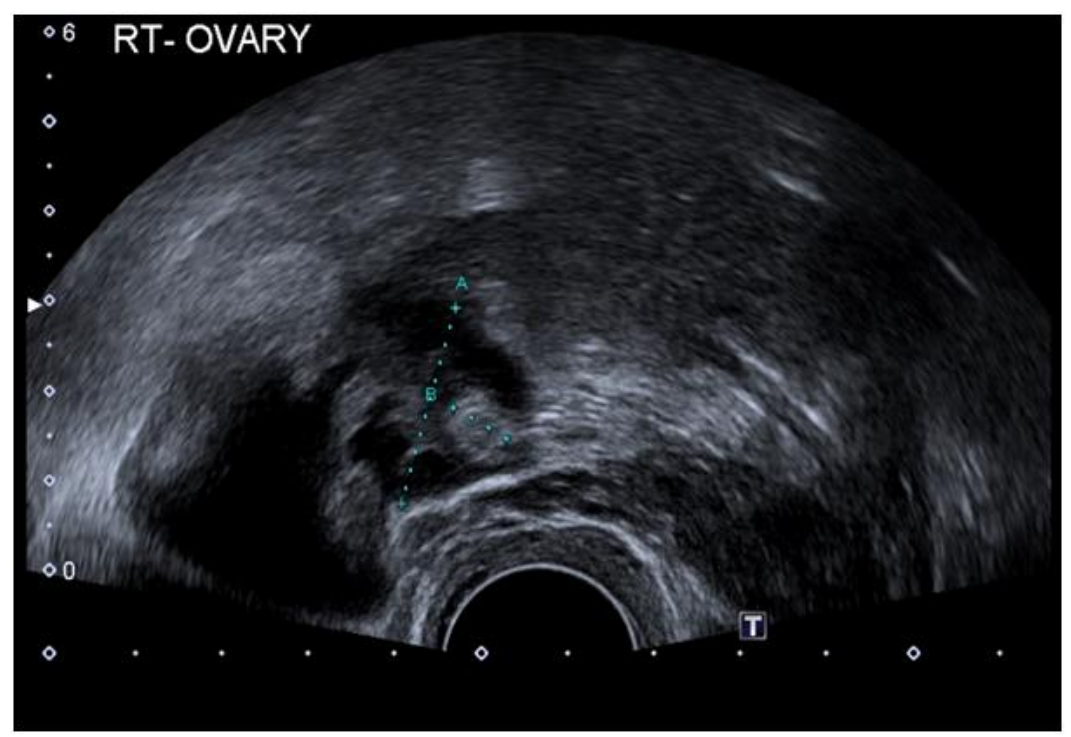

Figure 3: Further view of the small high reflectivity area on ultrasound.

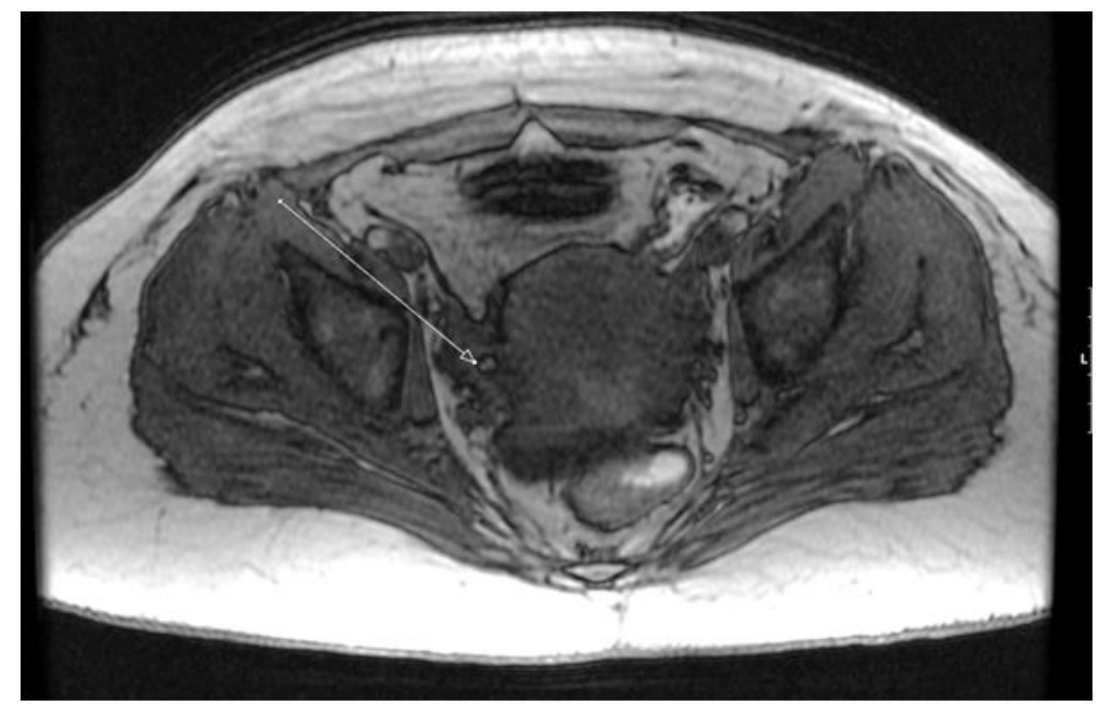

Figure 4: High T1 signal, suggestive of fat, within the right ovary.

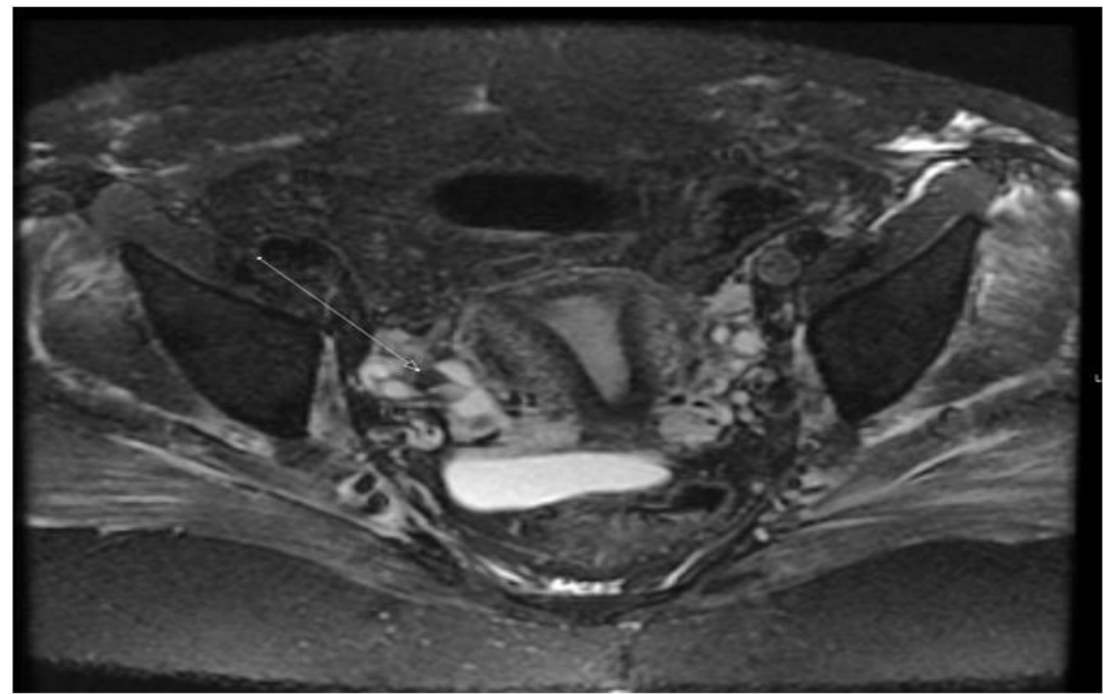

Figure 5: Low STIR signal, confirming fat, within the right ovary. 
http://www.tridhascholars.org | April-2020

\section{Discussion}

Various antibodies have been found to be the cause of inflammatory encephalitis that affects the limbic system. For example paraneoplastic causes include lung, testis, ovaries and breast cancers and non Hodgkin's lymphoma [1]. In our case a link between ovarian teratoma and anti-NMDAR encephalitis was proven. This is a very rare form and was first characterized by Dalmau et al. in 2008 [2].

Teratomas are tumours of germ cell origin. There is a recognized spectrum of disease from well differentiated, cystic benign lesions to solid and malignant lesions. Biopsies of the teratomas confirmed central nervous tissue and NMDAR in the case series by Dalmau et al. [2]. The antibodies can trigger symptoms that are recognized as anti- NMDAR encephalitis [3].

The initial presentation of anti-NMDAR encephalitis is a non-specific viral illness, including diarrhoea a, fever, nausea and vomiting [4]. After two weeks psychiatric symptoms are common, such as headaches, seizures, hallucinations and personality change [5].

CT or MRI brain is normally performed in the early stages to rule out an intracranial mass or haemorrhage as the cause for the symptoms. Lumbar puncture can also be performed and is initially normal with no evidence of a bacterial or viral cause. Investigating for a malignant cause, as was performed in our case is then carried out.

Treatment is supportive but in particular involves seizure management and removal of the underlying neoplasm. It can also involve immunotherapy.

\section{Consent Letter}

Not available.

\section{References}

1. Chua KH, Tan AWK, Lim SL, et al. (2017) Remove the ovarian tumor, cure the encephalitis: Anti-NMDAR encephalitis with ovarian teratoma in a tertiary hospital in Singapore. Journal of Medical Cases 8(2): 52-57.

2. Dalmau J, Gleichman AJ, Hughes EG, et al. (2008) Anti-NMDA-receptor encephalitis: case series and analysis of the effects of antibodies. The Lancet Neurology 7(12): 1091-1098.

3. Acién P, Acién M, Ruiz-Maciá E, et al. (2014) Ovarian teratoma-associated anti-NMDAR encephalitis: a systematic review of reported cases. Orphanet Journal of Rare Diseases 9(1): 157.

4. Jandu AS, Odor PM, Vidgeon SD (2016) Status epilepticus and anti-NMDA receptor encephalitis after resection of an ovarian teratoma. Journal of the Intensive Care Society 17(4): 346-352.

5. Braverman JA, Marcus C, Garg R (2015) Anti-NMDA-receptor encephalitis: A neuropsychiatric syndrome associated with ovarian teratoma. Gynecologic Oncology Reports 14: 1-3. 